\title{
Circuit Implementation, Operation, and Simulation of Multivalued Nonvolatile Static Random Access Memory Using a Resistivity Change Device
}

\author{
Kazuya Nakayama ${ }^{1}$ and Akio Kitagawa ${ }^{2}$ \\ ${ }^{1}$ Faculty of Medicine, Graduate School of Medical Science, Kanazawa University, 5-11-80, Kodatsuno, Kanazawa 920-0942, Japan \\ ${ }^{2}$ Faculty of Engineering, Graduate School of Natural Science \& Technology, Kanazawa University, Kakuma, Kanazawa 920-1192, Japan \\ Correspondence should be addressed to Kazuya Nakayama; knaka@kenroku.kanazawa-u.ac.jp
}

Received 21 June 2013; Revised 15 October 2013; Accepted 15 October 2013

Academic Editor: Ezz I. El-Masry

Copyright (C) 2013 K. Nakayama and A. Kitagawa. This is an open access article distributed under the Creative Commons Attribution License, which permits unrestricted use, distribution, and reproduction in any medium, provided the original work is properly cited.

\begin{abstract}
We proposed and computationally analyzed a multivalued, nonvolatile SRAM using a ReRAM. Two reference resistors and a programmable resistor are connected to the storage nodes of a standard SRAM cell. The proposed 9T3R MNV-SRAM cell can store 2 bits of memory. In the storing operation, the recall operation and the successive decision operation of whether or not write pulse is required can be performed simultaneously. Therefore, the duration of the decision operation and the circuit are not required when using the proposed scheme. In order to realize a stable recall operation, a certain current (or voltage) is applied to the cell before the power supply is turned on. To investigate the process variation tolerance and the accuracy of programmed resistance, we simulated the effect of variations in the width of the transistor of the proposed MNV-SRAM cell, the resistance of the programmable resistor, and the power supply voltage with $180 \mathrm{~nm} 3.3 \mathrm{~V}$ CMOS HSPICE device models.
\end{abstract}

\section{Introduction}

Power dissipation has been one of the most serious concerns in highly integrated CMOS logic circuits. For example, a leakage current's effect becomes dominant in the standby mode. One solution is to use nonvolatile memory, which has been proposed. Typical new types of memory include ferroelectric random access memory (FeRAM), magnetoresistive RAM (MRAM), phase change RAM (PRAM), and resistivity change RAM (ReRAM). A nonvolatile SRAM (NV-SRAM) has also been developed to mitigate restriction in program cycles and to improve the access time [1-5]. The component count of the NV-SRAM is large because the nonvolatile portion must be added to the SRAM portion.

The large resistivity change of ReRAM and PRAM is a superior characteristic and has been studied for multivalued nonvolatile memory [6-10]. For example, 16 separate states for multivalued storage were reported in [6]. ReRAM has been widely expected for use as the next generation of nonvolatile memory because of its superior characteristics, such as low-voltage operation, high-speed performance, and low power. This device has two switching modes: (i) unipolar switching, in which the device depends on the pulse width and amplitude of the applied voltage, and (ii) bipolar switching, in which the device depends on the polar character. In this paper, we used a bipolar switching device that made the best use of this proposed circuit. Figure 1 shows the definition of the set and reset operations for the bipolar switching device. Set is defined as an operation conducted to change from a high resistance state (HRS) to a low resistance state (LRS), and reset is an operation to change from LRS to HRS.

In this paper, we apply the multivalued storage technology to the NV-SRAM. The proposed multivalued nonvolatile SRAM (MNV-SRAM) can hold 2 bits (4 values) of memory in standby mode and acts as conventional SRAM in a normal (SRAM) operation. By replacing half of the conventional SRAM array with MNV-SRAM cells, NV-SRAM memory is realizable, as shown in Figure 2(a). The number of elements (transistor and resistor) required to hold 1 bit can be reduced using the proposed MNV-SRAM cells. Furthermore, when 


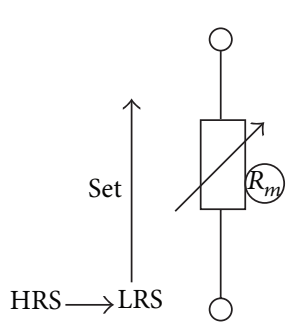

(a) Set operation

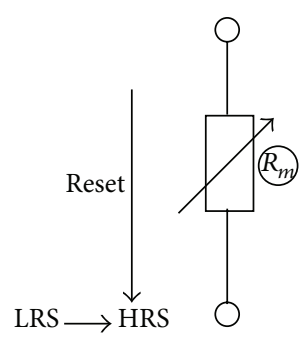

(b) Reset operation

FIgURE 1: Current flow of the set and reset operations of ReRAM (bipolar switching device).

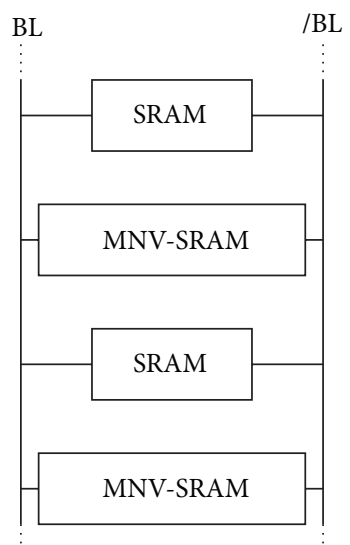

(a) SRAM + MN-VSRAM array

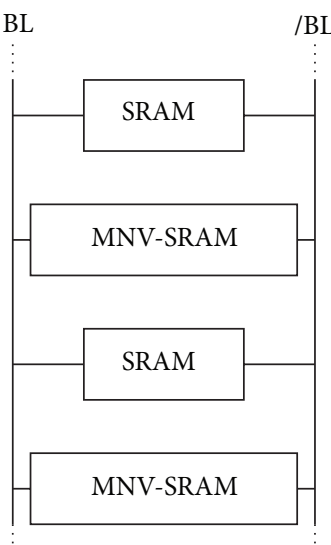

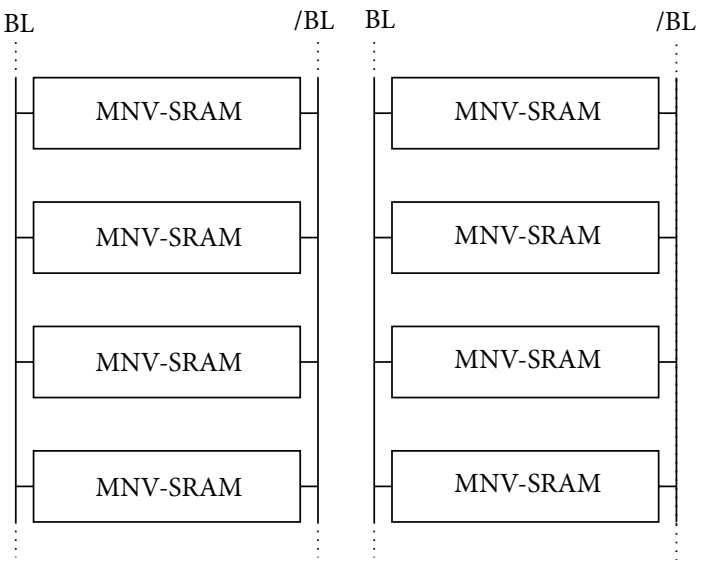

(b) MNV-SRAM array

FIgURE 2: Array structure. (a) Half of the array is SRAM. (b) All of the array is MNV-SRAM.

all of the cells are replaced with MNV-SRAM cells, as shown in Figure 2(b), the capacity becomes twice that of the conventional memory. We also propose a stable recall operation. The recall operation of conventional NV-SRAM with the programmable resistor is not very stable. It will become even more unstable since process variations increase along with the progress of scaling in CMOS technology.

In this paper, we describe the cell's design and operation. We also present the results from the HSPICE simulation of the proposed MNV-SRAM cell using a $180 \mathrm{~nm} 3.3 \mathrm{~V}$ CMOS device model.

\section{Cell Structure and Operation}

Figure 3 outlines the circuit topologies of conventional NV-SRAM and the proposed MNV-SRAM. The former 8 transistor 2 resistor (8T2R) cell is an improved cell for the conventional shadow RAM $[1,2]$ and has been applied to magnetic tunnel junctions (MTJs) [4]. The latter 9 transistor 3 resistor (9T3R) cell, which is added to the former 1 resistor $\left(R_{\text {refb }}\right)$ and 1 transistor (M9), also consists of standard SRAM portions and the nonvolatile memory portion. $R_{\text {ref }}, R_{\text {refa }}$, and $R_{\text {refb }}$ are fixed reference resistors and $R_{m}$ is a programmable resistor. These reference resistors and the programmable resistor are connected to the storage node ( $\mathrm{S} 1$ and S2) of a standard SRAM cell.
The proposed MNV-SRAM cell has two reference resistors $\left(R_{\text {refa }}\right.$ and $\left.R_{\text {refb }}\right)$. By having two reference resistors, the reference resistance can be changed to three values, $R_{\text {refa }}$, $R_{\text {refb }}$, and the parallel resistance of $R_{\text {refa }}$ and $R_{\text {refb }}\left(R_{\text {refa }} / / R_{\text {refb }}\right)$ as shown in Figure 4. For example, the reference resistance can be changed into $100 \mathrm{k} \Omega, 65 \mathrm{k} \Omega$, and $39 \mathrm{k} \Omega$, when $R_{\text {refa }}$ and $R_{\text {refb }}$ are set to $100 \mathrm{k} \Omega$ and $65 \mathrm{k} \Omega$, respectively. Therefore, 4 values (2 bits) can be determined as shown in Figure 4(b). It is not necessary to prepare three reference resistances $\left(R_{\text {refa }}\right.$, $R_{\mathrm{refb}}$, and $\left.R_{\text {refc }}\right)$. The resistance of $R_{m}$ depends on material (composition), size, and write/erase pulse conditions. It is necessary to define $R_{\text {refa }}$ and $R_{\text {refb }}$ according to the resistance distribution of $R_{m}$. For simplicity, in this paper, it is assumed that the distribution of $R_{m}$ is uniform from 0 to $150 \mathrm{k} \Omega$ and $R_{\text {refa }}$ and $R_{\text {refb }}$ are assumed to be fixed resistors which used high resistance polysilicon, and so forth.

The operation scheme, associated with control signals, and main current flows for the proposed MNV-SRAM are outlined in Figure 5. In order to read 2-bit information, the read operation is performed twice in the recall operation. The SRAM + MNV-SRAM array (Figure 2(a)) is used for introduction to the recall operation, and $R_{\text {refb }}$ is smaller than $R_{\text {refa }}$. The store operation is also introduced in this paper.

2.1. Recall Operation. It is assumed that $R_{m}$ stores (holds) 4 values ( 2 bits) in this paper. In the first recall operation, 


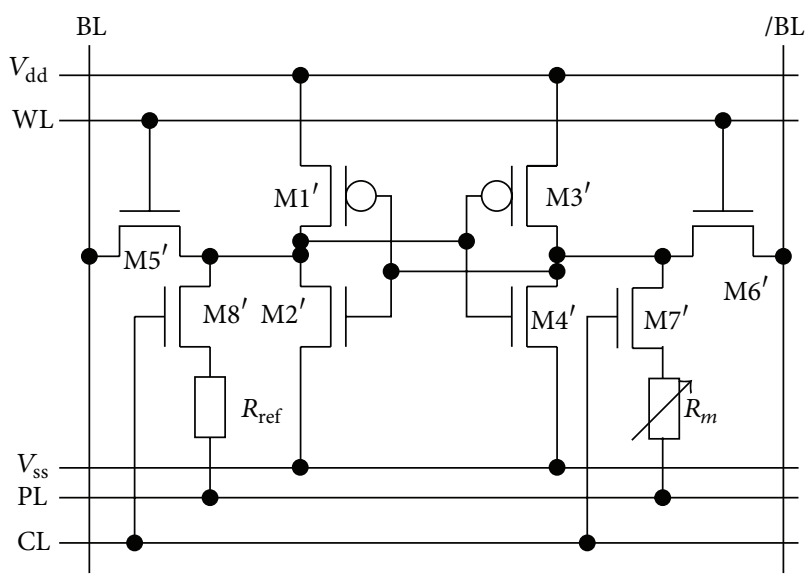

(a)

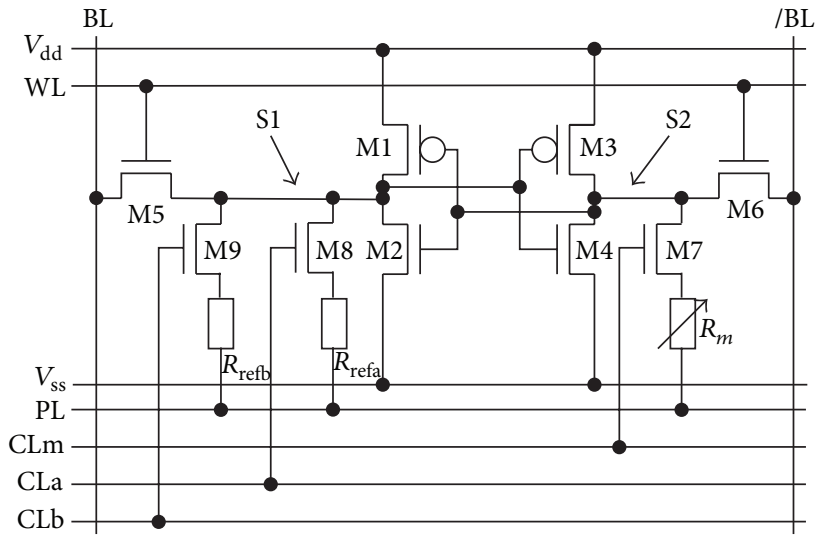

(b)

FIGURE 3: (a) Conventional NV-SRAM cell and (b) proposed MNV-SRAM.

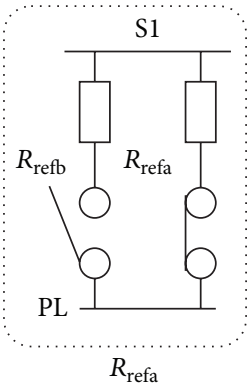

(a1)

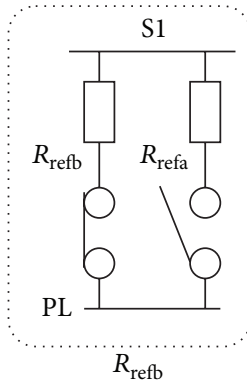

(a2)

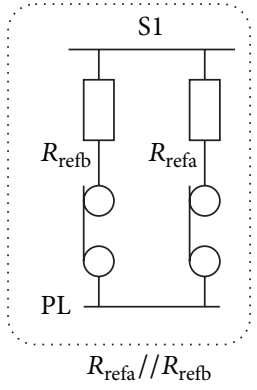

(a3)

(a)

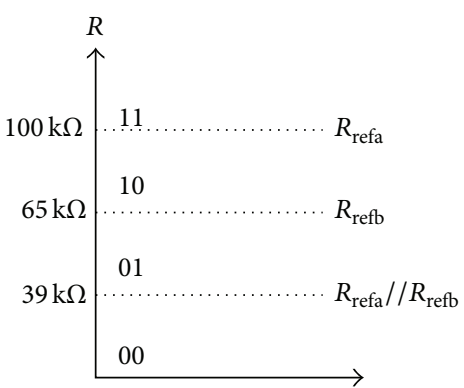

(b)

FIGURE 4: Reference resistances.

CLm, CLa, and CLb are set to "H," "L," and "H," respectively (M7 and M9 are turned on and M8 is turned off). Therefore, $R_{m}$ can be compared to $R_{\text {refb }}$ (Figure 4(a2)). During recall, transistors M7 and M9 are turned on. A certain current (or voltage) is applied from the precharge circuit (i) before/Cpm is set to the "L" state. The voltage of nodes $\mathrm{S} 1(V(\mathrm{~S} 1))$ and S2 $(V(\mathrm{~S} 2))$ in Figure 5(a) increases depending on $R_{\text {refb }}$ and $R_{m}$. When $R_{\text {refb }}$ is larger than $R_{m}, V(\mathrm{~S} 1)$ is lower than $V(\mathrm{~S} 2)$. Therefore, the information stored in $R_{m}$ can be recalled into the SRAM portion of MNV-SRAM after/Cpm is set to the "L" state (when the power supply for the cell is turned on). Then, the recalled data is moved from MNV-SRAM to SRAM using read and write circuits. The high-order bit is read out in this process.

In the second recall operation, both CLm and CLa are set to "H." CLb depends on the results of the first recall operation. When $R_{m}$ is smaller than $R_{\text {refb }}$, CLb is set to " $\mathrm{H}$." $R_{m}$ is compared to $R_{\text {refa }} / / R_{\text {refb }}$ (Figure $4(\mathrm{a} 3)$ ). On the other hand, when $R_{m}$ is larger than $R_{\text {refb }}$ CLb is set to "L." $R_{m}$ is compared to $R_{\text {refa }}$ (Figure $4(\mathrm{al})$ ). The low-order bit is read out in this process.

This recall step and read-out of 2-bit data are shown in Figure 6.
2.2. SRAM Operation. Normal SRAM operations can be performed by closing transistors M7, M8, and M9, as shown in Figure 5(b).

2.3. Store Operation. Figures 5(c) and 5(d) outline the store operation, which is performed just before power off. The information stored in SRAM (high-order bit) and (SRAM part of) MNV-SRAM (low-order bit) in Figure 5(a) is read first. The reference resistor is selected by this read information. For example, when the read information is " 01, " $R_{m}$

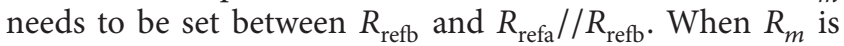
larger than $R_{\text {refb }}$, the set pulse needs to be applied to $R_{m}$. When $R_{m}$ is smaller than $R_{\text {refa }} / / R_{\text {refb }}$, the reset pulse needs to be applied to $R_{m}$ as shown in Figure 7 .

In order to compare $R_{m}$ with $R_{\text {refb }}$, CLm, CLa, and CLb are set to "H," "L," and " $H$," respectively. Then, the recall operation is carried out. When $R_{m}$ is larger than $R_{\text {refb }}$, node S2 is automatically set to " $\mathrm{L}$ " $(0 \mathrm{~V})$ in this recall operation. A set voltage (required for set operation) is applied from PL. After that, the set pulse is applied to $R_{m}$ in order to make $R_{m}$ have a lower resistance, as shown in Figure 5(c). On the other hand, when $R_{m}$ is smaller than $R_{\text {refb }}$, the $\mathrm{V}(\mathrm{S} 2)$ is set to " $\mathrm{H}$ " 


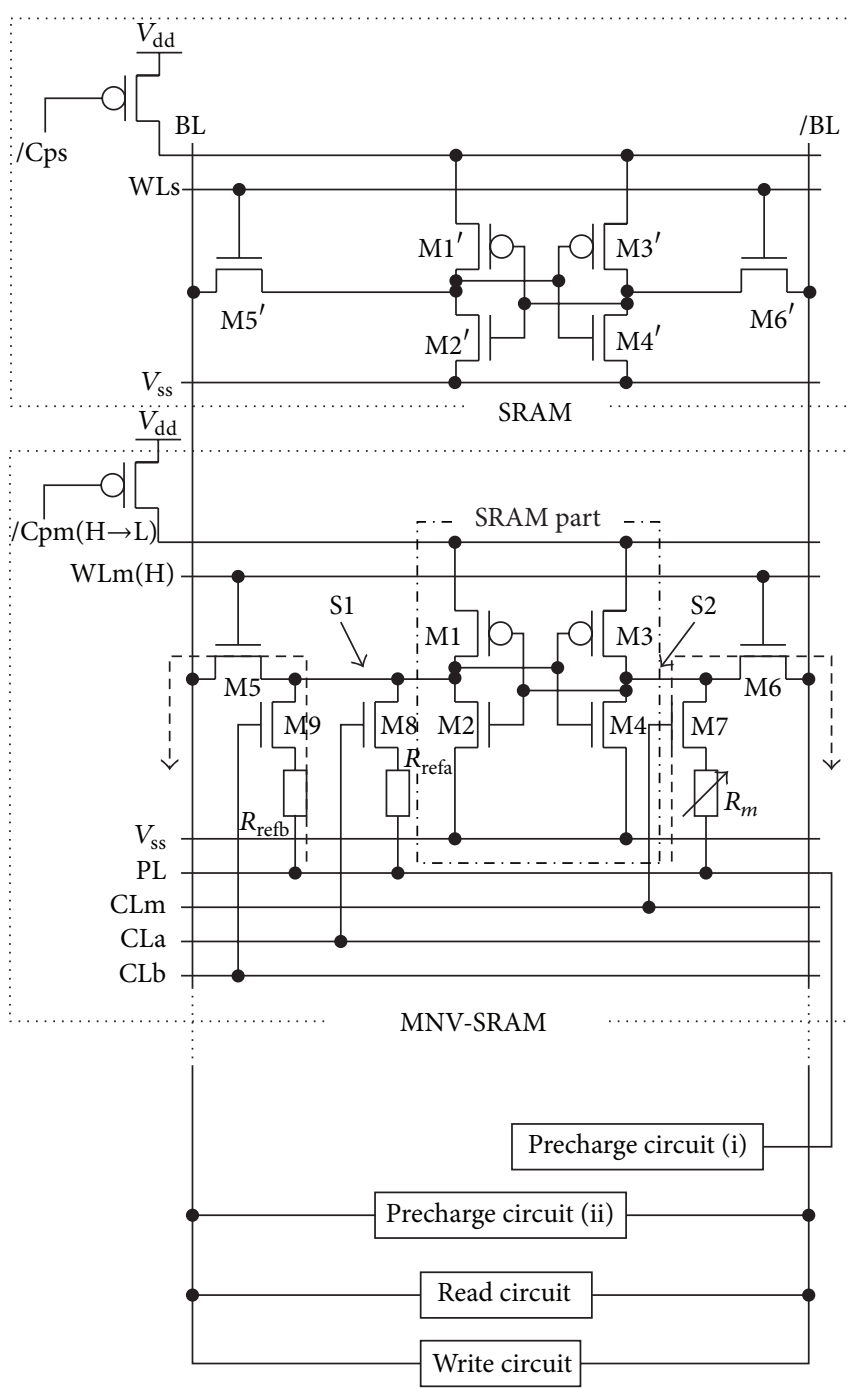

(a) Recall

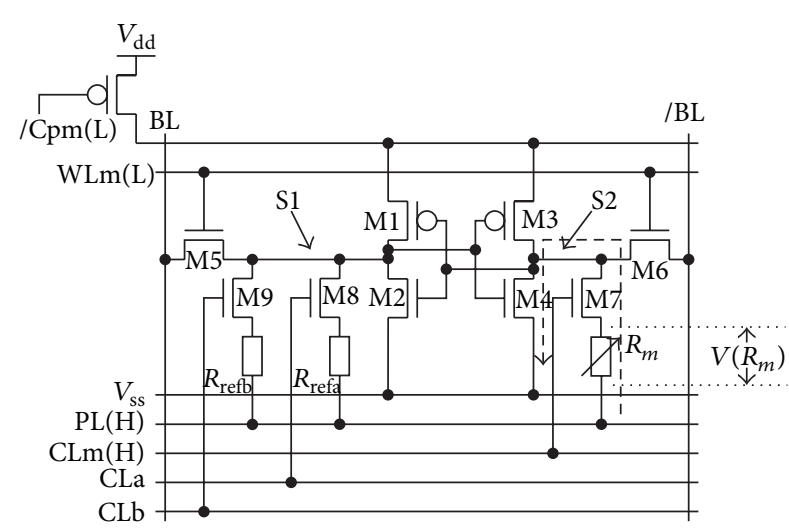

(c) Store (set)

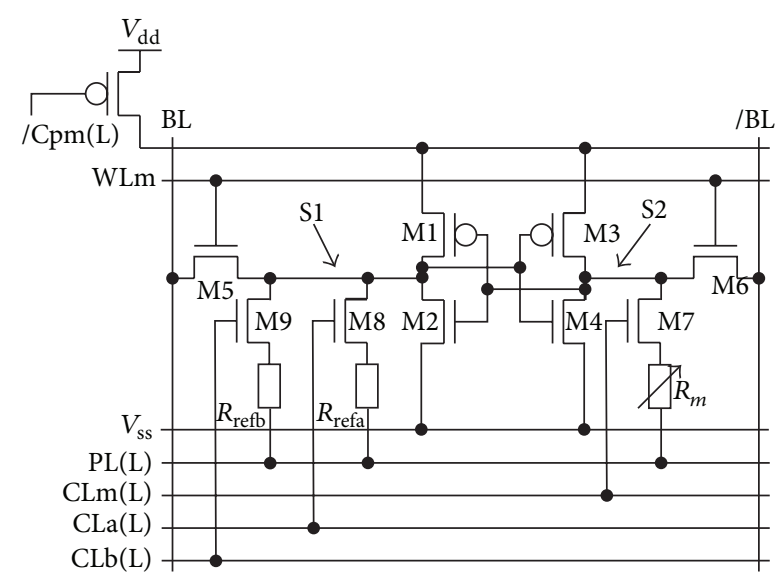

(b) SRAM

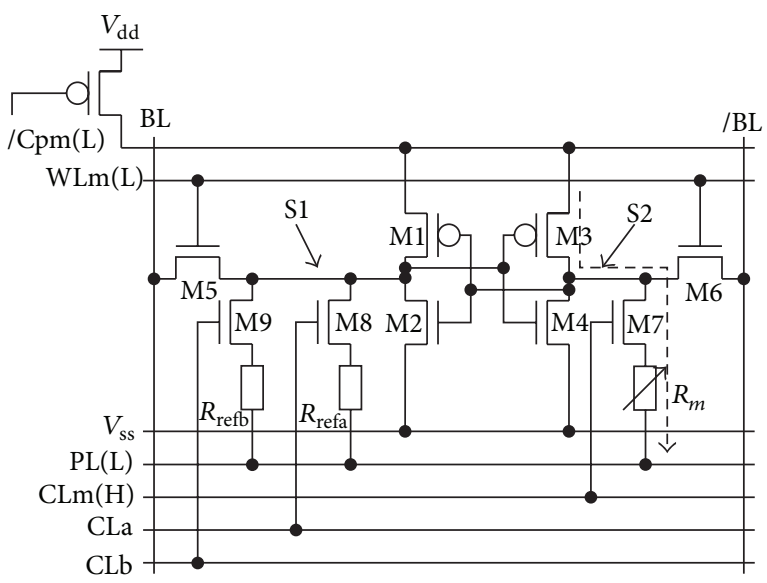

(d) Store (reset)

FIGURE 5: Operation scheme for MNV-SRAM. 


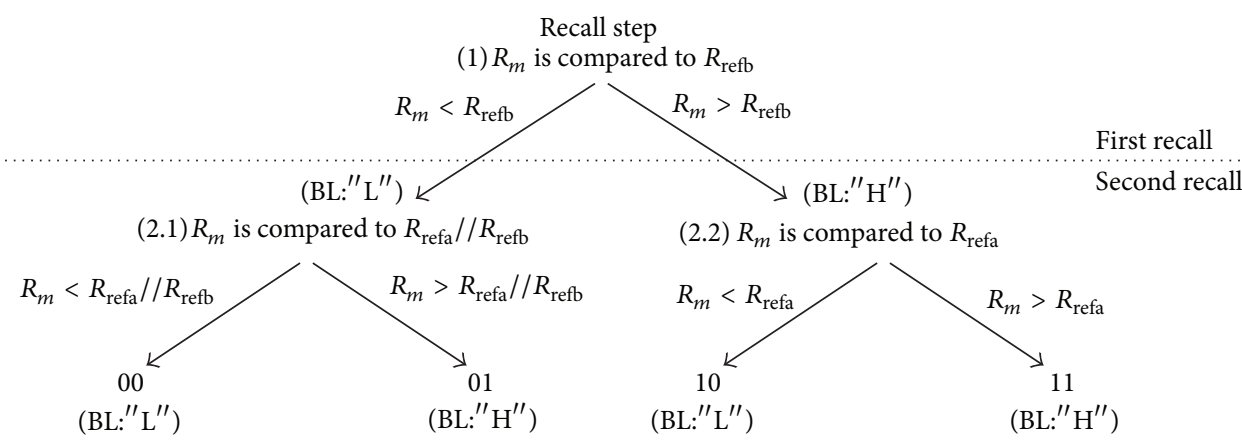

Figure 6: Recall step for MNV-SRAM.

TABLE 1: Relationship of $R_{m}$, nodes potential (S2 and PL), and applied pulse for the store operation.

\begin{tabular}{lcccc}
\hline & & $\mathrm{S} 2$ & $\mathrm{PL}$ & \\
\hline \multirow{2}{*}{ First store } & $R_{m}>R_{\text {refb }}$ & $\mathrm{L}$ & $\mathrm{H}$ & Set pulse \\
& $R_{m}<R_{\text {refb }}$ & $\mathrm{H}$ & $\mathrm{H}$ & - \\
Second store & $R_{\text {refa }} / / R_{\text {refb }}<R_{m}$ & $\mathrm{~L}$ & $\mathrm{~L}$ & - \\
& $R_{\text {refa }} / / R_{\text {refb }}>R_{m}$ & $\mathrm{H}$ & $\mathrm{L}$ & Reset pulse
\end{tabular}

TABLE 2: ReRAM device characteristics [11].

\begin{tabular}{lc}
\hline Set voltage & More than $2.2 \mathrm{~V}$ \\
Set transition time & $\sim 50 \mathrm{~ns}$ \\
Reset voltage & Less than $-1.4 \mathrm{~V}$ \\
Reset transition time & $\sim 50 \mathrm{~ns}$ \\
\hline
\end{tabular}

automatically. The current which flows through $R_{m}$ is zero (or a small value) because the voltage of both ends of $R_{m}$ is high (H).

Next, all of CLm, CLa, and CLb are set to " $\mathrm{H}$ " and the recall operation is carried out. $R_{m}$ is compared to $R_{\text {refa }} / / R_{\text {refb }}$. When $R_{m}$ is smaller than $R_{\text {refa }} / / R_{\text {refb }}$, node $\mathrm{S} 2$ is set to " $\mathrm{H}$ " automatically in this recall operation. Then, the PL is set to low voltage (or $0 \mathrm{~V}$ ). The reset pulse is applied to $R_{m}$, as shown in Figure 5(d). On the other hand, when $R_{m}$ is larger than $R_{\text {refa }} / / R_{\text {refb }}$, node S2 is set to "L" automatically. The current that flows through $R_{m}$ is zero (or a small value) because the voltage of both ends of $R_{m}$ is low.

The write pulse is not applied to $R_{m}$ when the value of $R_{m}$ is between $R_{\text {refa }} / / R_{\text {refb }}$ and $R_{\text {refb }}$ by using this method. The current flow directions of store (reset) and store (set) are opposite, as shown in Figures 5(c) and 5(d).

Table 1 shows the relationship of $R_{m}$, the nodes potential (S2 and PL), and the applied pulse.

\section{Simulation}

3.1. Simulation Condition. The device characteristics of the ReRAM are assumed in Table 2 [11]. These data are the set/reset conditions for a single-level cell using CoOx [11]. The resistance of the set state was $5 \mathrm{k} \Omega$ and the resistance of the reset state was reported to be within $10 \mathrm{k} \Omega$ and $150 \mathrm{k} \Omega$. Then, it was assumed that $R_{m}$ changes from $10 \mathrm{k} \Omega$ to $150 \mathrm{k} \Omega$,
TABLE 3: Simulation condition.

\begin{tabular}{lc}
\hline Simulator & HSPICE \\
Fabrication process & $180 \mathrm{~nm} \mathrm{CMOS}$ \\
Power supply & $3.3 \mathrm{~V}$ \\
Temperature & $300 \mathrm{~K}$ \\
\hline
\end{tabular}

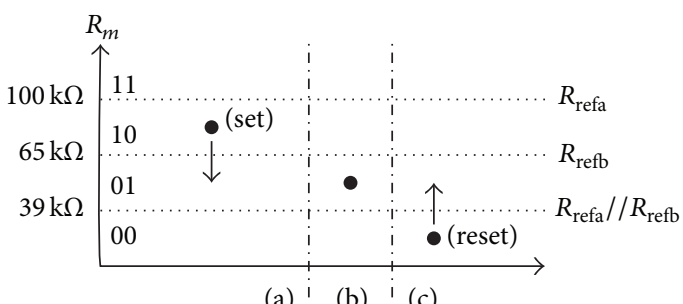

Figure 7: Write pulse required when "01" is stored in $R_{m}$.

and the set and reset voltages were assumed to be lower than the values shown in Table 2. 1024 cells were connected in a bit line (BL), as shown in Figure 2. Here, peripheral control circuits, such as address decoding and timing generation, are not included in our simulation condition. The wiring capacity and the wiring resistor are not included, either. The simulation conditions are listed in Table 3.

3.2. Recall Operation. Figures 8 and 9 show the simulation results for the recall operation. $R_{\text {refa }}$ and $R_{\text {refb }}$ were fixed at $100 \mathrm{k} \Omega$ and $65 \mathrm{k} \Omega$, respectively. In this case, the reference resistances $R_{\text {refa }}, R_{\text {refb }}$, and $R_{\text {refa }} / / R_{\text {refb }}$ are $100 \mathrm{k} \Omega, 65 \mathrm{k} \Omega$, and $39 \mathrm{k} \Omega$, respectively.

First, /Cpm was set to " $\mathrm{H}$ " and nodes $\mathrm{S} 1$ and S2 were charged once by $0 \mathrm{~V}$. Voltage (current) was applied to the BL, $/ \mathrm{BL}$, and PL using the precharge circuit (i) and (ii). A recall pulse of $600 \mathrm{mV}$ was produced from the precharge circuit (i) (at $41 \mathrm{~ns}$ ). When $R_{m}$ was $50 \mathrm{k} \Omega$, the voltage nodes $\mathrm{S} 1$ and $\mathrm{S} 2$ at $65 \mathrm{~ns}$ were $350 \mathrm{mV}$ and $570 \mathrm{mV}$, respectively. The difference voltage $V_{\mathrm{d} 1}(=V(\mathrm{~S} 1)-V(\mathrm{~S} 2)$ at $65 \mathrm{~ns})$ was $-220 \mathrm{mV}$. Since the voltage of node $\mathrm{S} 1$ was lower than that of node S2, after /Cpm was set to "L" (at $66 \mathrm{~ns}$ ), nodes S1 and S2 could be set to "L" and " $H$," respectively. Next, this recalled data was moved from MNV-SRAM to SRAM using read and write circuits. 


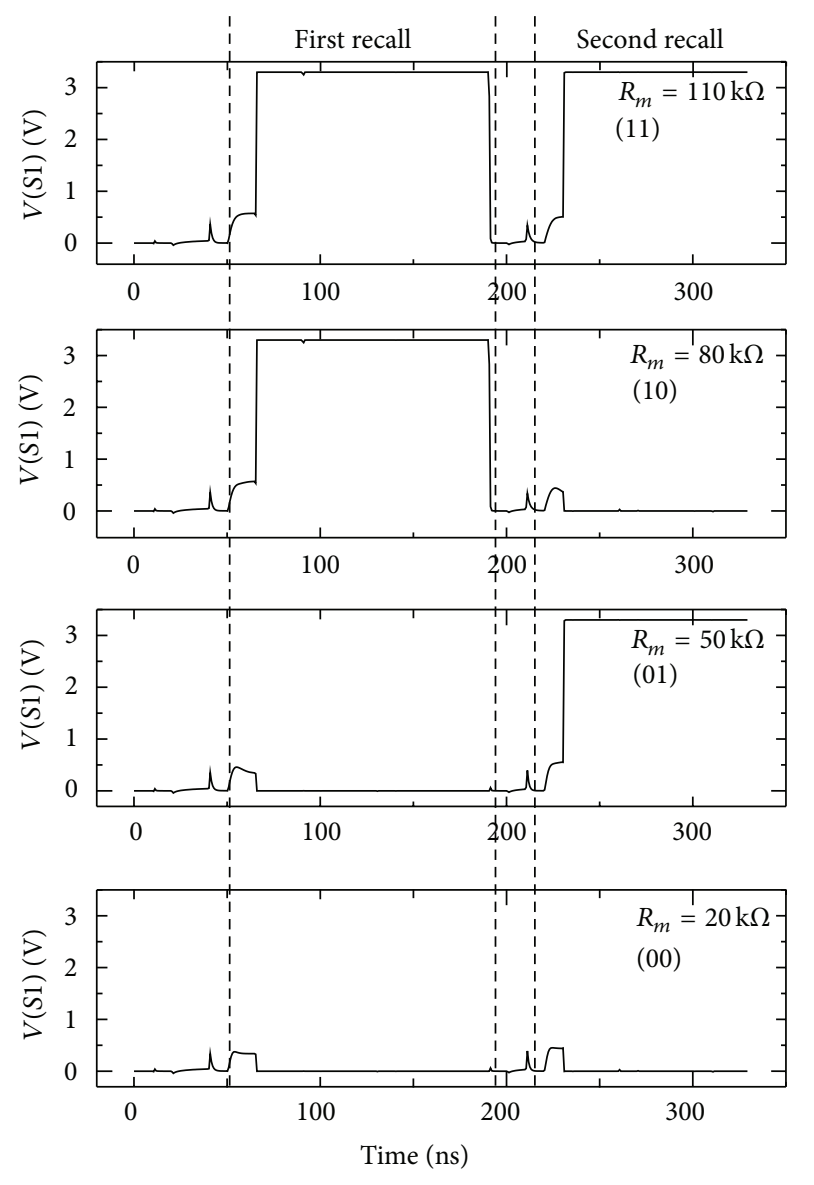

FIGURE 8: Simulation results for the recall operation. $R_{\text {refa }}$ and $R_{\text {refb }}$ were fixed at 100 and $65 \mathrm{k} \Omega$, respectively.

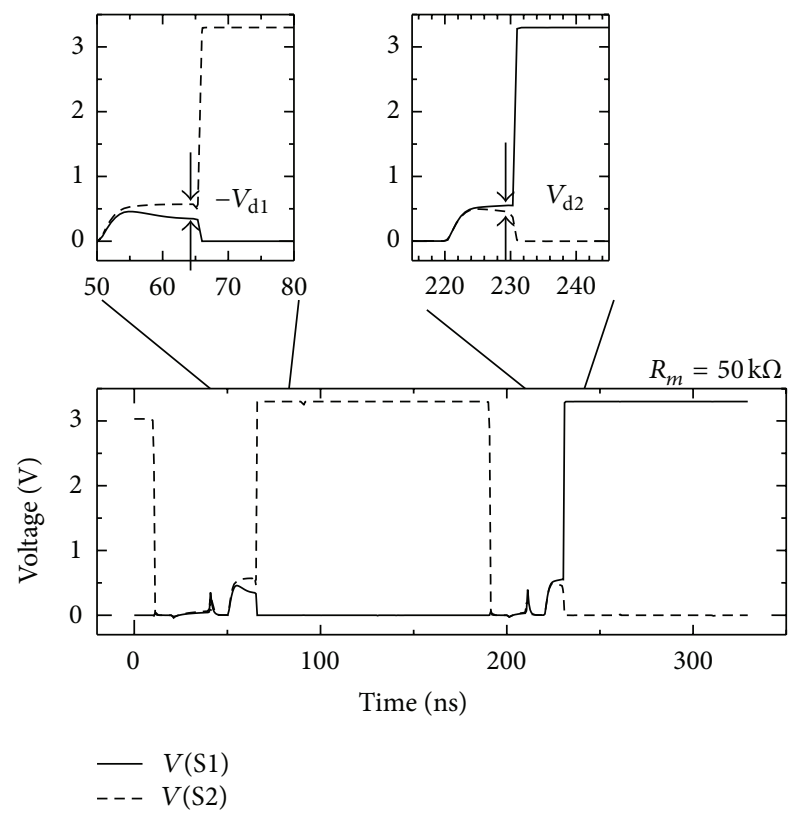

FIGURE 9: Simulation results for the recall operation. $R_{m}, R_{\text {refa }}$, and $R_{\text {refb }}$ were fixed at 50,100 , and $65 \mathrm{k} \Omega$, respectively.

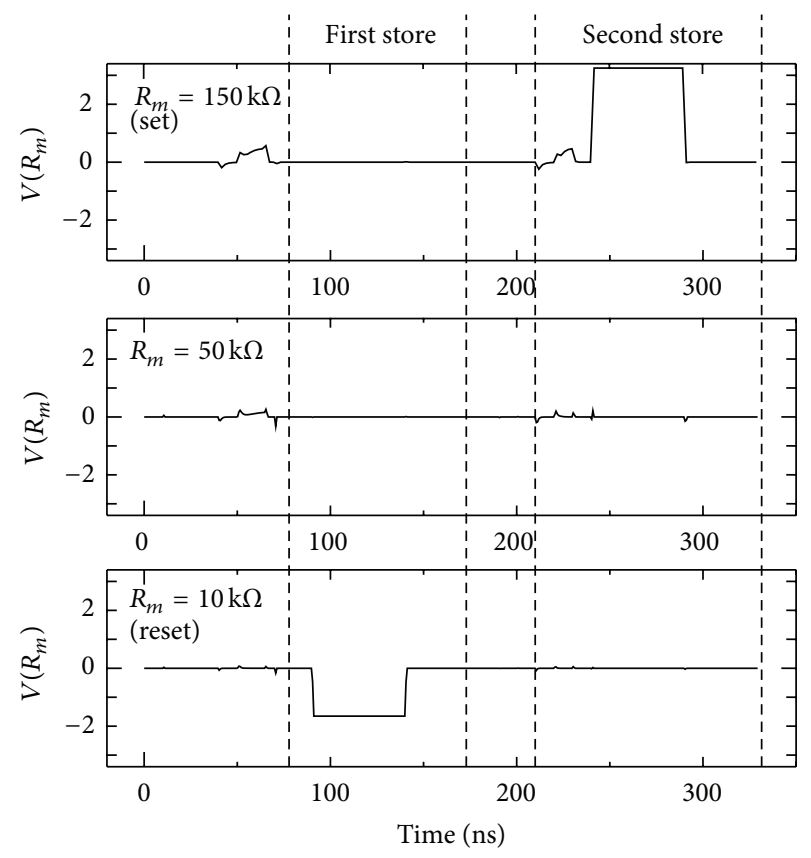

FIGURE 10: Simulation results for store operation. $R_{\text {refa }}$ and $R_{\text {refb }}$ were fixed at 100 and $65 \mathrm{k} \Omega$. In this case, the information " 01 " was stored in $R_{m}$.

Second, /Cpm was set to "H" and nodes S1 and S2 were again charged by $0 \mathrm{~V}$. The recall pulse of $600 \mathrm{mV}$ was applied to the $\mathrm{BL}$ and /BL using the precharge circuit at $220 \mathrm{~ns}$. When $R_{m}$ was $50 \mathrm{k} \Omega$, the voltage nodes $\mathrm{S} 1$ and $\mathrm{S} 2$ at $229 \mathrm{~ns}$ were $550 \mathrm{mV}$ and $460 \mathrm{mV}$, respectively. The difference voltage $V_{\mathrm{d} 2}$ $(=V(\mathrm{~S} 1)-V(\mathrm{~S} 2)$ at $229 \mathrm{~ns})$ was $90 \mathrm{mV}$. Since the voltage of node S1 was higher than that of node S2, after /Cpm was set to "L" (at $230 \mathrm{~ns}$ ), nodes $\mathrm{S} 1$ and $\mathrm{S} 2$ could be set to "H" and "L," respectively. Therefore, when $R_{m}$ is $50 \mathrm{k} \Omega$, the information "01" is recalled in this way.

3.3. Store Operation. When the information " 01 " is stored in $R_{m}, R_{m}$ needs to be set between $65 \mathrm{k} \Omega\left(=R_{\text {refb }}\right)$ and $39 \mathrm{k} \Omega$ $\left(=R_{\text {refa }} / / R_{\text {refb }}\right)$. There are three cases for the store operation, as shown in Figure 7. Figure 10 shows the simulation results for this store operation. It was confirmed that the circuits operated when the value of $R_{m}$ was from $10 \mathrm{k} \Omega$ to $150 \mathrm{k} \Omega$. When $R_{m}$ was 150 or $10 \mathrm{k} \Omega$, the set or reset pulse was applied to $R_{m}$, respectively. On the other hand, when $R_{m}$ was $50 \mathrm{k} \Omega$, the electric pulse did not need to be applied to $R_{m}$. The recall operation and the decision operation of whether or not the write pulse is required could be performed simultaneously. Therefore, the decision operation and circuit are not required when using this proposed scheme.

\section{Discussion}

4.1. Number of Elements of the Proposed Cell. Conventional MNV-SRAM cells consist of 8T2R, as shown in Figure 3(a) $[1,2,4]$. The proposed MNV-SRAM cell can hold 2 bits (4 values) of memory in standby mode by adding 3T3R to a 

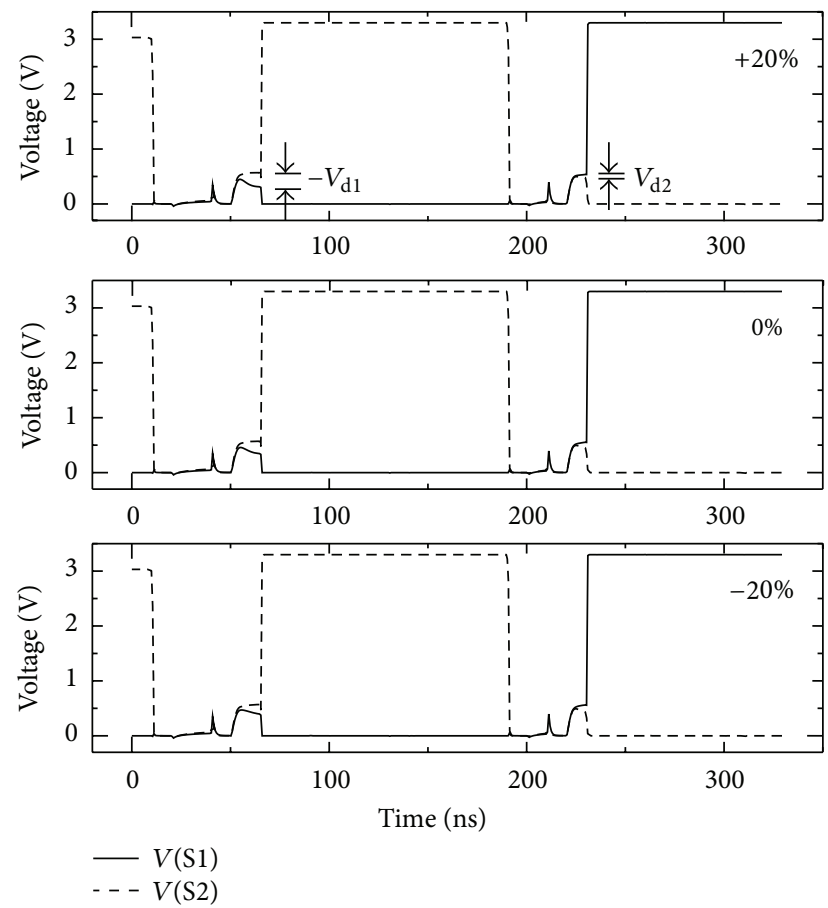

FIGURE 11: Effect of the channel width of the transistor on recall. Width of the channel of transistor M2 in Figure 3(b) varied from $-20 \%$ to $+20 \%$. $R_{\text {refa }}, R_{\text {refb }}$, and $R_{m}$ were fixed at 100,65 , and $50 \mathrm{k} \Omega$, respectively.

SRAM (6T) cell. In the array structure shown in Figure 1(a), 2 bits are recorded in 2 cells (SRAM + MNVSRAM, 6T + 9T3R). Therefore, the average number of elements required in order to memorize 1 bit of memory (ANE) is 7.5T1.5T, using the proposed MNV-SRAM cell. By using the proposed cell, the number of elements per cell can be reduced by $0.5 \mathrm{~T} 0.5 \mathrm{R}$.

If the number of reference resistors is increased, a cell can memorize much more information theoretically. For example, the cell with three reference resistors can encode 3 bits/cell, and the cell with four reference resistors can memorize 4 bits/cell. Therefore, ANE decreases with an increase in the quantity memorized per cell, as shown in Table 4. However, a read/write margin rapidly decreases with an increase in the quantity memorized per cell.

4.2. Recall Stability. One of the most serious problems with MNV-SRAM is the stability of recall. The data integrity of the proposed MNV-SRAM cell is dominated by recall since all resistance information must be properly regenerated to the corresponding SRAM and SRAM portion of MNV-SRAM. The recall operation is used also for the store operation. The effect of variations in device characteristics (device mismatch) on recall is described below. Figure 11 plots the effect of the channel width of the transistor on recall. For simplicity, we have only shown that the width of the channel of NMOS transistor M2 in Figure 3(b) varied by $20 \% . R_{\text {refa }}, R_{\text {refb }}$, and $R_{m}$ were fixed at 100, 65 and, $50 \mathrm{k} \Omega$, respectively. For example, when the width is narrowed by $-20 \%$, the $V_{\mathrm{d} 1}$ decreases $(-220 \mathrm{mV}(0 \%) \rightarrow-174 \mathrm{mV}(-20 \%))$ at $65 \mathrm{~ns}$. However,

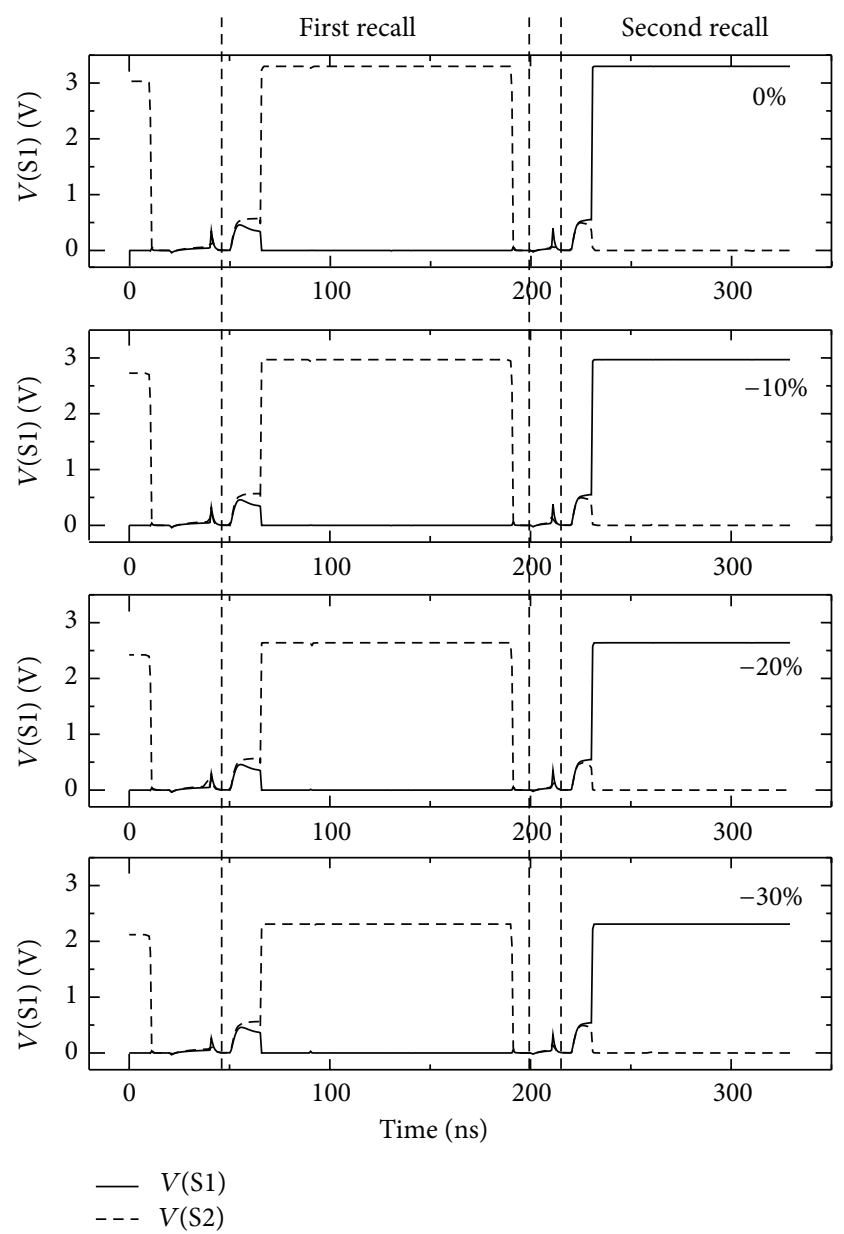

FIGURE 12: Effect of fluctuations on power supply voltage. Power supply voltage varied from $-30 \%$ to $0 \%$.

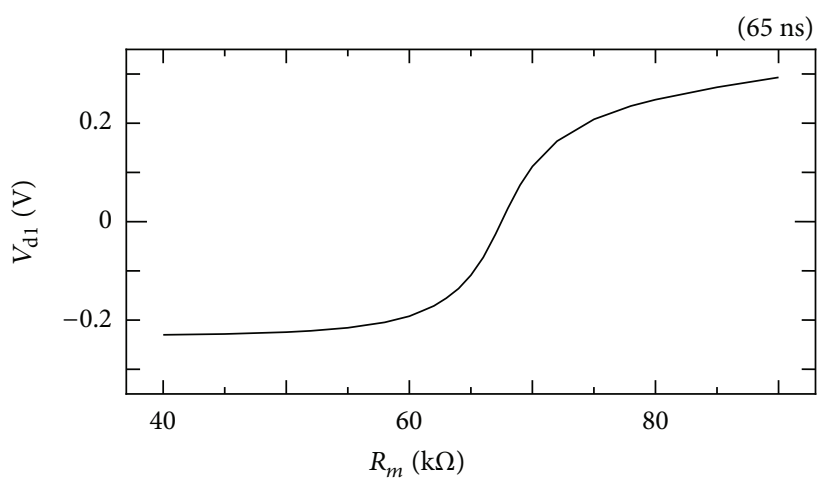

FIGURE 13: Effect of $R_{m}$ fluctuations. $V_{\mathrm{d} 1}$ shows the different voltage between the node S1 and S2 $\left(V_{\mathrm{d} 1}=V(\mathrm{~S} 1)-V(\mathrm{~S} 2)\right) . R_{m}$ was compared to $R_{\text {refb }}$, and $R_{\text {refb }}$ was fixed at $65 \mathrm{k} \Omega$. $R_{m}$ varied from 40 to $90 \mathrm{k} \Omega$.

the $V_{\mathrm{d} 2}$ increases slightly $(90 \mathrm{mV}(0 \%) \rightarrow 115 \mathrm{mV}(-20 \%))$ at $229 \mathrm{~ns}$. In all cases, the recall operation was performed correctly. However, the precharge operation is not used in the conventional recall operation [4]. The recall operation easily malfunctions without the precharge operation. For example, 


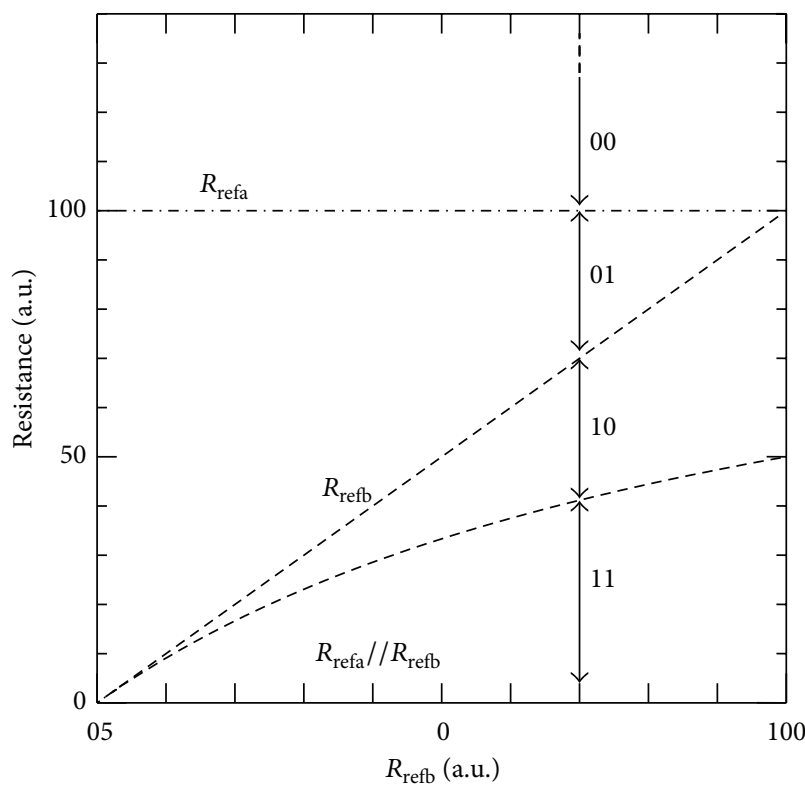

(a) Reference resistances

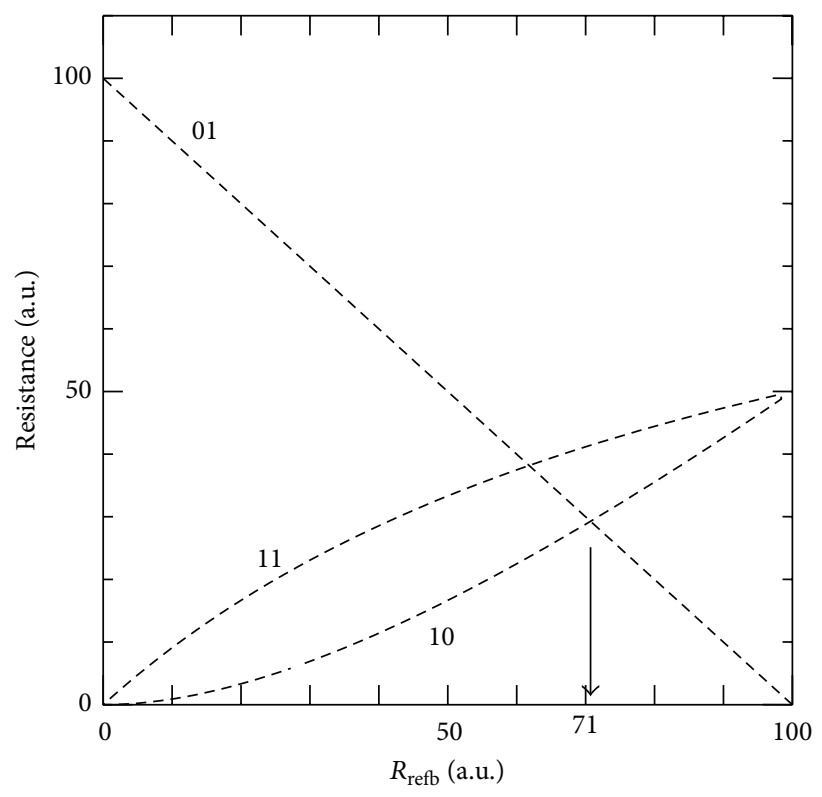

(b) Margins of reference resistances

FiguRE 14: (a) Reference resistances. $R_{\text {refa }}$ is fixed and $R_{\text {refb }}$ is changed from 0 to $100 . R_{\text {refa }} / / R_{\text {refb }}$ shows the parallel resistance of $R_{\text {refa }}$ and $R_{\text {refb }}$. (b) The difference between reference resistances (the amount of margins separating the states). 01 shows the difference between $R_{\text {refa }}$ and $R_{\text {refb }}$ $\left(R_{\text {refa }}-R_{\text {refb }}\right) .10$ shows " $R_{\text {refb }}-R_{\text {refa }} / / R_{\text {refb }}$ ". 11 shows the value of $R_{\text {refa }} / / R_{\text {refb }}$.

TABLE 4: The relation between the average number of elements required in order to memorize 1 bit (ANE) and the quantity memorized per cell (QMPC).

\begin{tabular}{lcccc}
\hline QMPC (bit/cell) & 1 & 2 & 3 & 4 \\
\hline ANE & 8 T 2 R & 7.5 T 1.5R & 7.33 T 1.33R & 7.25 T $1.25 \mathrm{R}$ \\
\hline
\end{tabular}

TABLE 5: Corner analysis of $V_{\mathrm{d} 1}$ during first recall.

\begin{tabular}{lccc}
\hline & & NMOS & \\
& Slow & Typical & Fast \\
\hline PMOS & & & \\
Slow & $-30 \mathrm{mV}$ & - & $-480 \mathrm{mV}$ \\
Typical & - & $-220 \mathrm{mV}$ & - \\
Fast & $-30 \mathrm{mV}$ & - & $-470 \mathrm{mV}$ \\
\hline
\end{tabular}

if the width of NMOS transistor M2 is narrowed by $1 \%$, the recall operation malfunctions without the precharge process.

Figure 12 shows the effects of power supply voltage fluctuations. The supply voltage varied from $-30 \%$ to $0 \%$. The recall operation was correctly executed for the supply voltage fluctuations. However, a drop in the supply voltage to $-30 \%$ reduced the voltage difference to $30 \mathrm{mV}(-220 \mathrm{mV}$ $\rightarrow-190 \mathrm{mV}$ at $65 \mathrm{~ns}$ ).

Tables 5 and 6 list the dependencies of the $V_{\mathrm{d} 1}$ and $V_{\mathrm{d} 2}$ on transistor models. In this case, $R_{\text {refa }}, R_{\text {reff }}$, and $R_{m}$ are fixed at 100,65 , and $50 \mathrm{k} \Omega$, respectively. The $V_{\mathrm{d} 1}$ and $V_{\mathrm{d} 2}$ strongly depend on the characteristic of NMOS.

Figure 13 shows the effect of $R_{m}$ fluctuations. $V_{\mathrm{d} 1}$ showed different voltages between the nodes $\mathrm{S} 1$ and $\mathrm{S} 2\left(V_{\mathrm{d} 1}=V(\mathrm{~S} 1)-\right.$ $V(\mathrm{~S} 2))$. $R_{m}$ was compared to $R_{\text {refb }}$, and $R_{\text {refb }}$ was fixed at
TABLE 6: Corner analysis of $V_{\mathrm{d} 2}$ during second recall.

\begin{tabular}{lccc}
\hline & \multicolumn{3}{c}{$\begin{array}{c}\text { NMOS } \\
\text { Typical }\end{array}$} \\
\hline PMOS & & & Fast \\
Slow & $13 \mathrm{mV}$ & - & $380 \mathrm{mV}$ \\
Typical & - & $90 \mathrm{mV}$ & - \\
Fast & $14 \mathrm{mV}$ & - & $380 \mathrm{mV}$ \\
\hline
\end{tabular}

$65 \mathrm{k} \Omega$. $R_{m}$ varied from 40 to $90 \mathrm{k} \Omega$. The threshold resistance was $67 \mathrm{k} \Omega$ and it was a little bigger than $R_{\text {refb. }}$. It seems that the threshold resistance shifted, since the number of resistors (FETs) connected to $\mathrm{S} 1\left(R_{\text {refa }}\right.$ and $\left.R_{\text {refb }}\right)$ and $\mathrm{S} 2\left(R_{m}\right)$ differed. The recall operation is easy when the absolute value of $V_{\mathrm{d} 1}$ is large. If the absolute value of $V_{\mathrm{d} 1}$ must be $100 \mathrm{mV}$ or more, $R_{m}$ must be smaller than $65 \mathrm{k} \Omega$ or larger than $70 \mathrm{k} \Omega$ in this study. If the circuits are optimized, these points could improve.

4.3. Reference Resistor. In order to enlarge a read margin, it is necessary to enlarge the reference resistance differences. Figure 14 shows the reference resistances and the differences between the reference resistances. $R_{\text {refa }}$ was fixed at 100 and $R_{\text {refb }}$ was changed from 0 to 100 . The differences of the reference resistances reached a maximum by $R_{\text {refa }}^{2}=2 R_{\text {reff }}^{2}$. For example, the values of $R_{\text {refa }}$ and $R_{\text {refb }}$ were set to $100 \mathrm{k} \Omega$ and $71 \mathrm{k} \Omega$, respectively. Then, the value of $R_{\text {refa }} / / R_{\text {refb }}$ was set to $42 \mathrm{k} \Omega$.

4.4. Energy Break Even Time. The energy break even time (EBT) [12] is also important for MNV-SRAM. The EBT 
depends on write/recall energy, the leakage current of FETs, and so forth. However, it is difficult to estimate the EBT because the energy-consuming write/verify cycles are usually required in multivalued nonvolatile memory. Therefore, the EBT also depends on the number of write/verify cycles. Although it was a rough estimate, we estimated the EBT by the following easy model. (i) The set/reset condition in [11] (Table 2) and its behavior model [13] were used. (ii) The write energy was assumed to be the average of set and reset energy as reported by Kawabata et al. [11]. (iii) Write and recall cycles were only one cycle each and the verifying cycle was zero. It was estimated that the EBT was $0.5 \mathrm{~s}$ in this condition. The EBT would improve when low power operating devices (materials) were used and/or the circuits were optimized.

\section{Conclusion}

We proposed a MNV-SRAM using a ReRAM. The proposed 9T3R MNV-SRAM cell can store 2 bits of memory and achieve stable recall against process variations and extended program cycles.

In order to realize the stable recall operation, a certain current (or voltage) is applied to the cell before the power supply is turned on. The voltage of nodes S1 and S2 of cells increases depending on $R_{\text {refb }}$ and $R_{m}$. When $R_{\text {refb }}$ is larger than $R_{m}, V(\mathrm{~S} 1)$ is lower than $V(\mathrm{~S} 2)$. Therefore, the information stored in $R_{m}$ can be stably recalled when the power supply for the cell is turned on.

To investigate the stability of recall, we simulated the effect of variations in the width of the transistor of the proposed NV-SRAM cell, the resistance of the sample, and the power supply voltage. For example, when the channel width of the transistor of the cell is narrowed by $-20 \%$, the $V_{\mathrm{d} 1}$ decreases (from $-224 \mathrm{mV}(0 \%)$ to $-174 \mathrm{mV}(-20 \%)$ ) at $65 \mathrm{~ns}$. On the other hand, the $V_{\mathrm{d} 2}$ increases slightly (from $92 \mathrm{mV}$ $(0 \%)$ to $115 \mathrm{mV}(-20 \%))$ at $229 \mathrm{~ns}$. Be that as it may, the recall operation was performed correctly.

In the store operation, the recall operation and the decision operation of whether or not the write pulse is required can be performed simultaneously. The decision operation and circuit are not required when using this proposed scheme.

\section{Acknowledgments}

This work was supported by VLSI Design and Educational Center (VDEC), The University of Tokyo, in collaboration with Cadence Design Systems, Inc., Synopsys, Inc., and Mentor Graphics, Inc. The VLSI chip in this study has been fabricated in the chip fabrication program of VDEC, the University of Tokyo, in collaboration with Rohm Corporation and Toppan Printing Corporation. This work was also supported by a Grant-in-Aid for Scientific Research (C) (23560391).

\section{References}

[1] S. Eaton, D. Butler, M. Parris, D. Wilson, and H. Mcnellie, "A ferroelectric nonvolatile memory," in Proceedings of the
International Solid-State Circuits Conference(ISSCC '88), pp. 130-131, 1988.

[2] S. Masui, T. Ninomiya, T. Ohkawa et al., "Design and application of ferroelectric memory based nonvolatile SRAM," IEICE Transactions on Electronics, vol. E87-C, no. 11, pp. 1769-1776, 2004.

[3] M. Takata, K. Nakayama, T. Izumi, T. Shinmura, J. Akita, and A. Kitagawa, "Nonvolatile SRAM based on phase change," in Proceedings of the 21st IEEE Non-Volatile Semiconductor Memory Workshop (NVSMW'06), pp. 95-96, February 2006.

[4] S. Yamamoto and S. Sugahara, "Nonvolatile static random access memory using magnetic tunnel junctions with currentinduced magnetization switching architecture," Japanese Journal of Applied Physics, vol. 48, no. 4, Article ID 043001, 2009.

[5] A. Kitagawa and K. Nakayama, "Phase change nonvolatile SRAM and Resister," in Proceedings of the 21th Symposium on Phase Change Optical information Storage (PCOS '09), pp. 33 36, 2009.

[6] J. Maimon, E. Spall, R. Quinn, and S. Schnur, "Chalcogenidebased non-volatile memory technology", in Proceedings of the IEEE Aerospace Conference, vol. 5, pp. 2289-2294, March 2001.

[7] K. Nakayama, M. Takata, T. Kasai, A. Kitagawa, and J. Akita, "Pulse number control of electrical resistance for multi-level storage based on phase change," Journal of Physics D, vol. 40, no. 17, article 009, pp. 5061-5065, 2007.

[8] Y. Yin, K. Ota, T. Noguchi, H. Ohno, H. Sone, and S. Hosaka, "Multilevel storage in $\mathrm{N}$-doped Sb2Te3-based lateral phase change memory with an additional top TiN layer," Japanese Journal of Applied Physics, vol. 48, no. 4, Article ID 04C063, 2009.

[9] S. Lee, J.-H. Jeong, T. S. Lee, W. M. Kim, and B.-K. Cheong, "Bias polarity dependence of a phase change memory with a Ge-doped SbTe: a method for multilevel programing," Applied Physics Letters, vol. 92, no. 24, Article ID 243507, 2008.

[10] Y. Han, K. Cho, and S. Kim, "Characteristics of multilevel bipolar resistive switching in $\mathrm{Au} / \mathrm{ZnO} / \mathrm{ITO}$ devices on glass," Microelectronic Engineering, vol. 88, no. 8, pp. 2608-2610, 2011.

[11] S. Kawabata, M. Nakura, S. Yamazaki et al., "CoOx-RRAM memory cell technology using recess structure for $128 \mathrm{Kbits}$ memory array," in Proceedings of the IEEE International Memory Workshop (IMW '10), pp. 16-19, May 2010.

[12] K. Usami and N. Ohkubo, "A design approach for fine-grained run-time power gating using locally extracted sleep signals," in Proceedings of the 24th International Conference on Computer Design (ICCD '06), pp. 155-161, October 2006.

[13] T. Handa, Y. Yoshimoto, K. Nakayama, and A. Kitagawa, "Novel power reduction technique for ReRAM with automatic avoidance circuit for wasteful overwrite," Active and Passive Electronic Components, vol. 2012, Article ID 181395, 11 pages, 2012. 

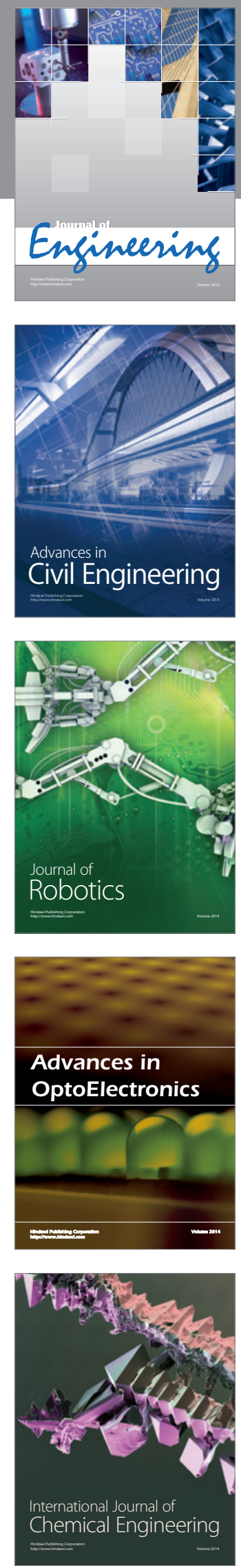

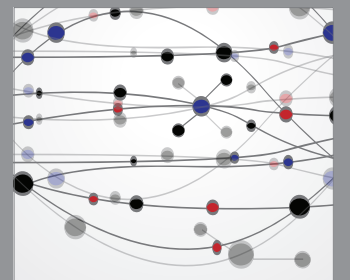

The Scientific World Journal
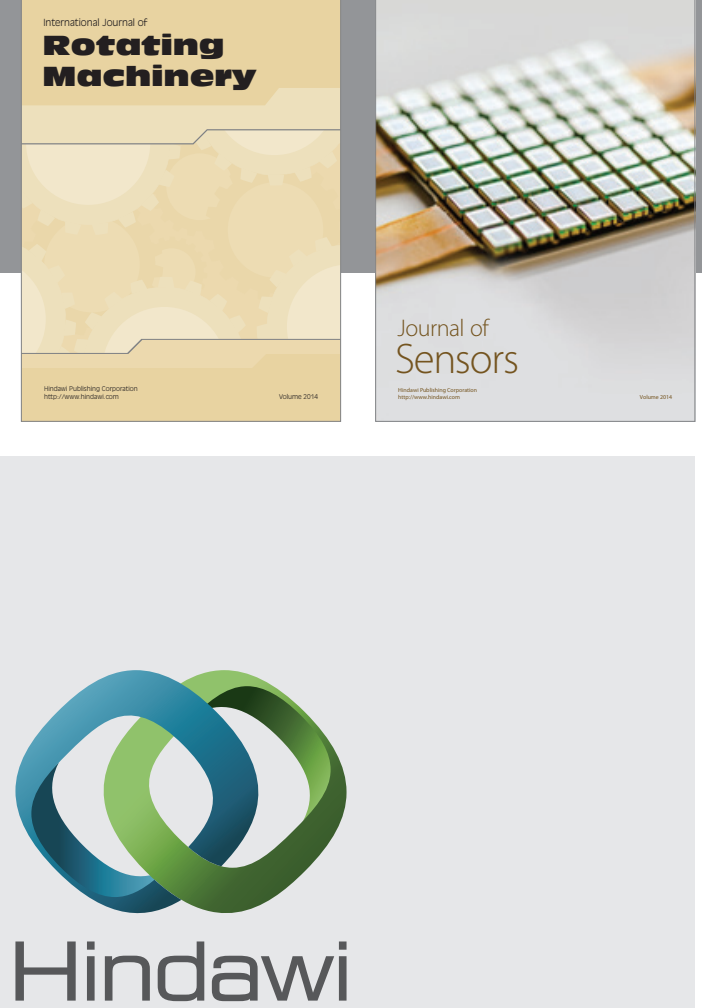

Submit your manuscripts at http://www.hindawi.com
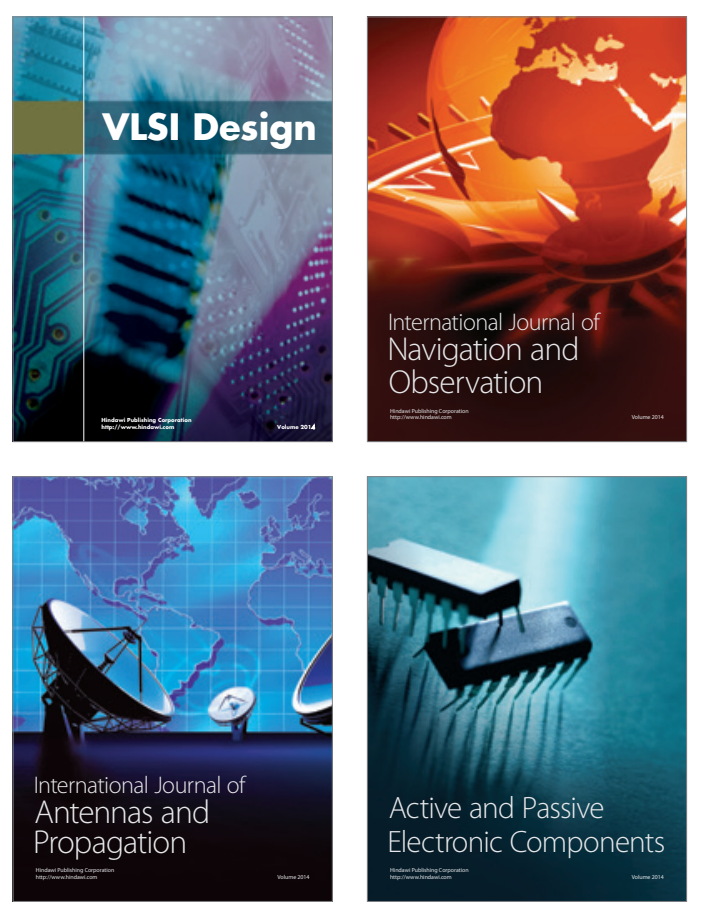
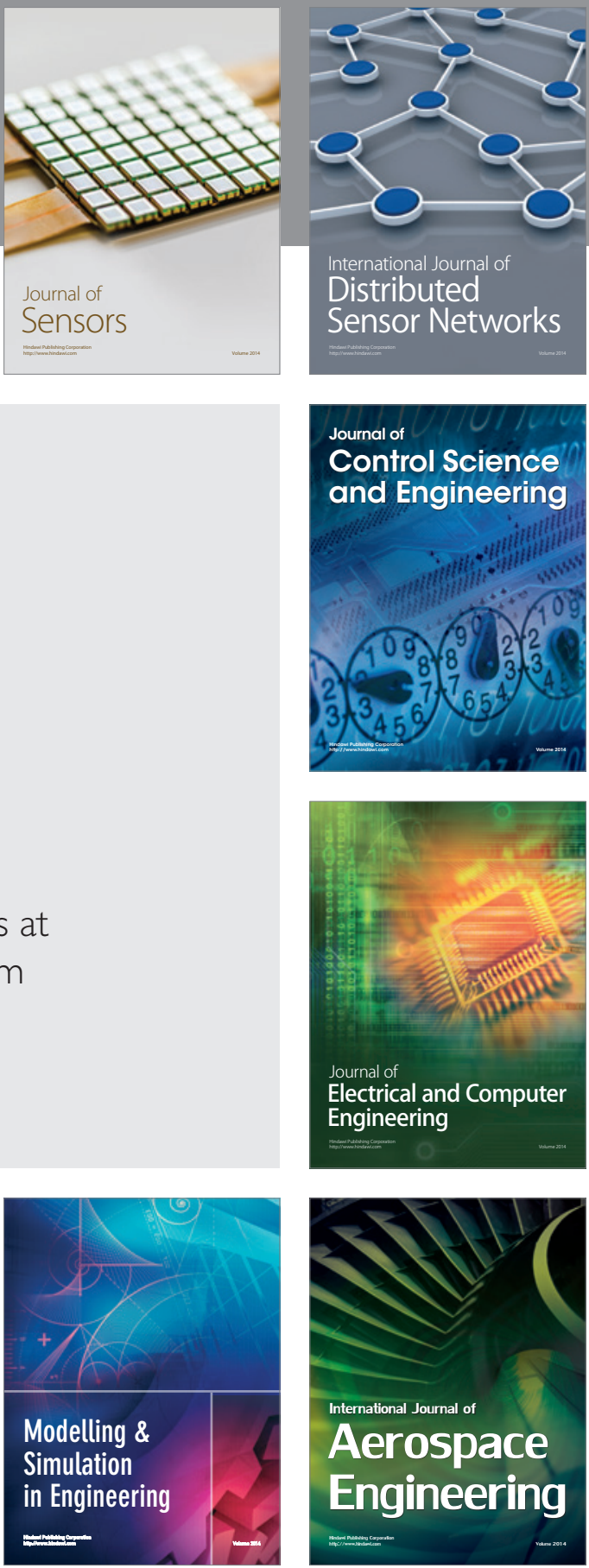

Journal of

Control Science

and Engineering
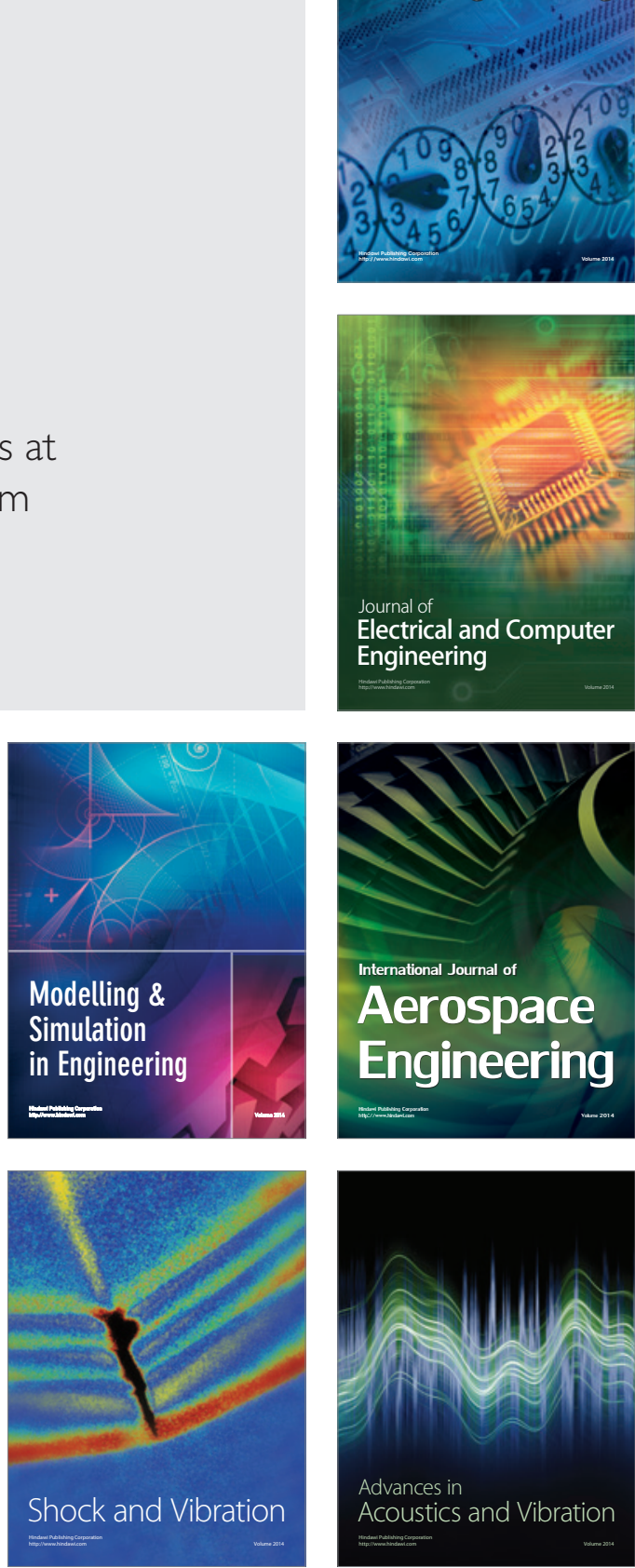